\title{
Pathophysiology and Treatment Options in Obstructive Sleep Apnoea: A Review of the Literature
}

\author{
Lars Rasmusson $^{1 *}$, Armin Bidarian ${ }^{2}$, Lars Sennerby ${ }^{1}$, Gareth $_{\text {Scott }}{ }^{3}$ \\ ${ }^{1}$ Department of Oral and Maxillofacial Surgery, The Sahlgrenska Academy, University of Gothenburg, Gothenburg, Sweden; \\ ${ }^{2}$ Department of Otolaryngology, The Sahlgrenska Academy, University of Gothenburg, Gothenburg, Sweden; ${ }^{3}$ Department of Or- \\ thopaedic Surgery, Barts and The London School of Medicine and Dentistry, Queen Mary, University of London, London, UK. \\ Email: *lars.rasmusson@gu.se
}

Received July $5^{\text {th }}$, 2012; revised August $10^{\text {th }}, 2012$; accepted September $20^{\text {th }}, 2012$

\begin{abstract}
Obstructive sleep apnoea, OSA, is formally defined as an apnoea-hypopnoea index (AHI) of at least 5 episodes/hour (mild). Obesity, alcohol, smoking and sedatives may contribute to the development of OSA, as well as tonsilar hypertrophy and maxillary and/or mandibular retrognathia. Endocrine conditions such as hypothyroidism and acromegaly have also been reported associated with OSA. Except for excessive daytime sleepiness, headaches and irritability, OSA may lead to hypertension, heart failure, MI and stroke. Management involves reduction of predisposing factors such as obesity and alcohol. Treatment with continuous positive airway pressure (CPAP) is currently most common and best documented. Also uvulopalatopharyngeal surgery and so called phase II surgery, which means advancement of the jaw(s) with orthognatic surgical methods are used. Treatment with dental appliances is getting increasingly popular but the long term outcome remains debatable.
\end{abstract}

Keywords: Airway Pathophysiology; Obstructive Seep Apnoea; Review

\section{Introduction}

Obstructive sleep apnoea is variously referred to in the literature by the following terms in a synonymous manner: obstructive sleep apnoea syndrome (OSAS), obstructive sleep apnoea/hypopnoea syndrome (OSAHS) and the shorter obstructive sleep apnoea (OSA). In this review only the latter will be used. The objectives were to review published information and data on the pathophysiology, epidemiology, investigations and recommended treatment of obstructive sleep apnoea. References were collected from an online PubMed survey in which over 13,800 papers were found (March, 2011). The survey was then refined and original papers and reviews were inspected to ensure they fulfilled the objectives above. Older papers were generally avoided and the majority of references used in this review were published from 1990-2011. Only internationally recognized journals with referee system were used. In total, 81 papers were used as references.

Sleep apnoea is a medical problem characterized by interrupted breathing during sleep. The affected period of time may be 10 seconds or more, during which breathing stops, followed by arousal and/or 3\% drop in oxygen saturation. Hypopnoea occurs when there is a $50 \%$ de-

\footnotetext{
*Corresponding author.
}

crease in airflow for at least 10 seconds, followed by arousal and/or 3\% drop in saturation. It is the oxygen desaturation leading to arousal from sleep that causes day time sleepiness rather than the apnoea itself.

There are three types of sleep apnoea: central sleep apnoea (CSA), obstructive sleep apnoea (OSA) and mixed sleep apnoea. During sleep, the respiratory centre stimulates the respiratory muscles of the chest to take a breath. Central sleep apnoea occurs when the CNS does not send the signal to the muscles to take a breath. Obstructive sleep apnoea occurs when the brain sends the instruction to the muscles and the muscles make an effort to take a breath, but they are unsuccessful because the airway becomes obstructed and prevents an adequate flow of air. Mixed sleep apnoea, occurs when there is both central sleep apnoea and obstructive sleep apnoea.

OSA is a common disorder and occurs if the upper airway closes during inspiration and/or expiration. It results from collapse of the pharyngeal airway during sleep. The mechanisms and cause of this occlusion are multifactorial, but in the end a result of the upper airway anatomy interacting with physiological mechanisms during sleep [1], e.g. reduced or absent dilator muscle control during sleep. There are, historically, two schools of thoughts describing the pathophysiology, the active vs the passive theories. The "active" theory was first pre- 
sented by Weitzman and co-workers and describes in an animal model, the spasmodic closure of the lateral pharyngeal walls and velopharynx timed at the end of expiration [2]. The muscle contraction persists during inspiration and does not return to the relaxed phase until the test object aroused from sleep. However, electromyography in humans has failed to demonstrate any muscle activity during airway closure and therefore the other "passive" theory has gained more acceptance [3]. The "passive" theory implies that no active neuromuscular contraction of the pharyngeal muscles is needed to close the airway. Instead, obstruction is the result of the tongue falling backwards together with impaired or complete loss of dilating activity of the pharyngeal muscles during sleep [4]. Most likely, both theories are at least partly true since OSA is a multifactorial phenomenon. The human airways have a complicated task. The ventilation should constantly be maintained together with speech and phonation. Additionally, the upper airway (larynx) should allow for alimentation down through the oesophagus without interference with the airway.

\section{Anatomical Abnormalities and the Physiology of OSA}

Neuromuscular factors are of importance as well as structural abnormalities which predispose to OSA $[5,6]$. Airway shape and size are critical to the air flow. In children, hypertrophy of adenoids and tonsils is the main cause but also craniofacial disorders such as micrognathia and soft tissue anomalies have an impact on OSA in childhood [7]. No single factor has been identified in the same way in adults. There are several anatomical factors contributing to the obstructive periods. Obesity is one important factor and it is well known that excessive weight direct increases the risk and severity of OSA due to augmentation of fat around the neck and airway. But obesity, which will be further discussed, also has an effect on metabolism and lung volume which might be of greater importance for the severity of OSA than the local changes in the upper airway.

\subsection{Head-Neck Abnormalities}

Maxillofacial variables associated with snoring and sleep apnoea include a relatively long distance from the mandibular plane to the hyoid bone and mandibular and/or maxillary retrognathia. An inferiorly displaced hyoid position may interfere with breathing since it would signify abnormalities in airway length and increased tongue size. Sometimes the relations between the jaws are within normal limits (orthognathia) but there is a downward bi-maxillary rotation resulting in increased vertical height of the lower third of the face. This in turn will lead to a longer posterior airway and also increased cervical angu- lation, all potentially contributing to OSA. It has since long been an established fact that small mandibles would have most impact on the severity of OSA, but in fact, Dempsy and co-workers have shown that in non-obese patients, abnormalities in maxillary position, size and especially width were the main contributors to OSA [8]. Accordingly, it could be anticipated that the obstruction is primarily a result of a disorder of the maxilla and less the mandible. Also nasal obstruction and sometimes complete blockage have an impact on the presence and severity of OSA. An obstructed nose will increase the tendency for breathing through the mouth which subsequently destabilizes the lower pharyngeal airway by displacement of the hyoid. The pharyngeal soft tissues contribute to OSA and the size of the tissue is of importance. In the supine position the tongue falls posteriorly and counteracted by the tone of the genioglossal muscle. Volumetric studies have shown that tongue size is a major predictor of OSA together with weak lateral walls in the pharynx, resulting in collapse and difficulties in reopening the airways [9]. The tongue is larger and the soft palate is both longer and wider in OSA patients compared to healthy individuals. The neuromuscular tone of the airway contributes to the balance of forces and patency of the airway which will be further discussed in the following paragraphs. Activation of the upper airway muscles is suppressed by various sedative medications and alcohol, which possibly explains why snoring and OSA worsen after intake of alcohol or diazepam. Interestingly, this is not the case for diaphragmatic activity.

\subsection{Structure and Forces}

A so called balance of forces model allow for an accurate description of how multiple variables alter the upper airway size. Upper airway size is determined by both dilating and collapsing forces. Dilating forces include upper airway muscle tone, mechanical force of the airway wall structure and positive intraluminal airway pressure. Collapsing forces include tissue mass, surface adhesive forces and negative intraluminal pressures. The resulting difference in these forces is what acts on the wall of the upper airway [1]. This means that when distending forces increase the airway, size also increases and when the forces decrease, the airway size subsequently decreases. A soft and collapsible tube could be used as a model to study the different forces acting upon the walls of the human upper airway - the supraglottic pharynx. When the patient is awake there is a low negative inspiratory intraluminal pressure combined with a large upper airway area, the nose, which results in unaffected flow. During sleep there is still a patent and stable upper airway in non-apnoeic people because the transmural pressure is higher than both inspiratory and expiratory pressures. On the other hand, apnoeic people have a smaller 
and less stable upper airway resulting in transmural pressures during sleep becoming lower than pressures in both upwards and downwards directions [1]. When the transmural pressure is falling and becomes lower than the closing pressure of the airway during inspiration, the airway collapses and no flow occurs. Using the same model one could say that snorers have an intermediate condition where the expiratory pressure is higher than the transmural pressure, but the inspiratory pressure is less. Flutter will be the result caused by a choke point of the airway exposed to alternating positive and negative transmural pressure. In OSA, increased resistance and the collapse of the upper airway happen not only during inspiration but also during expiration. Expiratory collapse is essentially a static process. The maximum airway collapse occurring at end expiration and precedes dynamic obstruction. The relatively low lung volume of air at the end of expiration + passive declining pressures in the airway lumen will contribute to the collapse. Expiratory obstruction will make breathing more tiresome since the apnoeic individual needs to overcome the effects of positive airway pressure (PEEP) [10]. The size of the airway is dependent on the ventilatory cycle and in OSA, the cross section area increases during early inspiration but is getting much smaller during the end of expiration. There is a progressive narrowing of the airways and finally the narrowing either completely obstruct it or results in a critical size where the previously mentioned negative pressure creates airway closure at the following inspiration. Not only the size, but also the shape of the airway may contribute to the pathogenesis of OSA. Some individuals with normal airway size and normal muscle activity nonetheless develop OSA. Patients with OSA tend to have elliptical airways with the long axis of the ellipse oriented along the anteroposterior axis and this orientation may negatively affect upper airway muscle function [11].

\subsection{Vascular Effects and Lung Volume}

Blood volume changes in the head-neck region can affect upper airway size. This has been shown in a study by altering leg elevation and the corresponding changes in central venous pressure. Its direct relationship to OSA is not known but it is considered an indirect proof of physical contribution [12]. A more direct effect on the pharyngeal airway size is lung volume. Increased lung volumes increase pharyngeal size and decreased volumes contribute to pharyngeal collapse. It has been demonstrated that changes are a mechanical effect of tracheal and thoracic traction. Thoracic traction (or tracheal tug) markedly influences pharyngeal size and patency and is mediated through the mediastinum, intrathoracic pressure and the trachea. Passive tracheal traction likely alters pharyngeal collapsibility by influencing tension and stability on the pharyngeal wall [13].

\subsection{Control of Ventilation}

The sleep levels REM and, NREM, as well as awakeness differ in arousal mechanisms and ventilatory sensitivity to hypoxia and hypercapnia. Central ventilatory control is destabilized by changes in level of consciousness. Hypercapnic drive is the most tightly controlled response and is also the primary determinant for ventilatory control. This drive decreases from wakefulness to NREM and REM sleep, and may result in hypoventilation during sleep if not compensated. In a person without OSA, hypoxic ventilatory drive also decreases from wake to NREM and REM. During sleep, ventilation is mediated by carbon dioxide and chemical control through both the central nervous system and indirectly through the carotid bodies. Cyclic (oscillating) arousals affect ventilation and ventilatory drive because of the influence of the CNS on the responsiveness of the afferent receptors which in turn affect airway stability. Arousal acutely activates and stabilizes the upper airway muscles in sleep. Brief awakenings rapidly shift central $\mathrm{CO}_{2}$ sensitivity lower to the level of wakefulness. This increases ventilation and quickly reduces $\mathrm{CO}_{2}$. This variable in respiratory control is chemoresponsiveness to hypercapnia and hypoxia and is termed controller gain. The higher the controller gain, the brisker the responsiveness to hypercapnia. The effectiveness of the level of ventilation to eliminate $\mathrm{CO}_{2}$ is termed plant gain. According to Woodson and Franco (2007), both types of gain are important in ventilatory stability [1].

\subsection{Obesity}

OSA is a disorder whose prevalence is linked to the epidemic of obesity in Western society. Obesity and particularly central adiposity are potent risk factors due to pressure to the airways. But obesity will also increase pharyngeal collapsibility through mechanical effects on pharyngeal soft tissue and lung volume and through central nervous system acting proteins that in turn may affect airway neuromuscular control [14].

Leptin, a $16 \mathrm{kDa}$ protein (167 amino acids) is playing a key role in regulating energy intake and energy expenditure, including appetite and metabolism. Leptin is known to suppress the appetite, but many obese people have built up a resistance to the appetite-suppressing effects of leptin. It is an adipose derived hormone, meaning that the more obese a person is the more leptin is produced. The level of circulating leptin is directly proportional to the total amount of fat in the body. In addition to white adipose tissue, leptin can also be produced in the ovaries, skeletal muscles, placenta, liver and bone marrow [15]. 
Leptin and various cytokines increase $\mathrm{CO}_{2}$ response and may contribute to central ventilatory sensitivity. However, due to the high levels of leptin and that obese people have an increased resistance to this protein, the obstructive breathing may worsen. This phenomenon has been proven correct in animal models [16], but need further evaluation in humans.

\section{The Apnoea Hypopnoea Index}

The apnoea hypopnoea index (AHI), shows apnoea and hyponoea episodes per hour of sleep. The AHI is probably the most useful and objective way of classifying the severity of OSA. Obstructive Sleep Apnoea Syndrome is a term that indicates a clinical entity defined by a high AHI in conjunction with hypersomnolence or related symptoms in daytime function. Recommended diagnostic criteria for OSA syndrome include an AHI of 5 or more determined by overnight monitoring and evidence of disturbed sleep, daytime sleepiness or other daytime symptoms. The classification has three levels; mild (AHI 5 - 14), moderate (AHI 15 - 30) and severe (AHI > 30) [17].

\section{Epidemiology}

OSA is the most common cause of excessive day time sleepiness. In most epidemiological studies, OSA is defined by the number of apnoea or hypopnoea episodes per hour as described above. Probability studies in adults shows an estimated prevalence for OSA of at least mild level, ranging from $3 \%-28 \%$ and for a moderate level ranging from $1 \%-14 \%[18,19]$. When only those studies with in-laboratory polysomnography conducted on large samples are compared, the prevalence estimates are in closer agreement. Results from cohort studies using 2stage stratified probability sampling, the estimates in men and women with a body mass index from 25 - 28 showed that roughly 1 in 5 adults have at least mild level of OSA and 1 in 15 has at least moderate level [20-22].

\subsection{Impact of Age}

Durán and co-workers, [22,23] have in two cohort studies found age differences in the prevalence of OSA. For ages 71 - 100 years, the prevalence of AHI $>5$ was $80 \%$ for women and $81 \%$ for men (95\% confidence interval). When compared with an age group of 30 - 70 years, the prevalence in older age group was about 3 times higher. In the younger group, 30 - 70 years, there was an increase in prevalence with age with an odds ratio of 2.2 for each 10 years. Similarly, in the Sleep Heart Health Study, [24] the proportion of people with an AHI > 15 events per hour was approximately 1.7 -fold higher in older (60 - 99 years) compared to younger (40 - 60 years) participants. It has been hypothesized that female sex hormones protect against OSA but a study of body fat distribution and sleep apnoea severity showed no difference between premenopausal and postmenopausal women [25].

\subsection{Impact of Gender}

There is a higher prevalence of OSA reported for men compared with women, with a male to female ratio of between 2.3:1 [26,27]. In two studies comparing snoring and AHI between middle-aged men and women, Ferini-Strambi and co-workers [28,29] showed using a home monitoring equipment that $7 \%$ of the females snored more than $50 \%$ of the night. The corresponding figure for males was similar, $8 \%$. But when comparing AHI, the differences between the sexes increased. The number of females with an AHI > 10 was $9 \%$ and the number who had an AHI $>20$ was $2 \%$. The corresponding figures for males were respectively $15.5 \%$ and $5 \%$. According to these studies of middle aged European populations, OSA (moderate to severe) was 2.5 times more common in males and snoring was a stronger predictor for OSA in males. A retrospective review of 830 consecutively diagnosed OSA patients showed the male:female ratio increasing from 2.3:1 for mild cases to 7.9:1 for severe cases [30].

\subsection{Snoring and OSA in Children}

As mentioned before, in children, hypertrophic adenoids and tonsils are the main causes of snoring and OSA but also maxillofacial disorders such as micrognathia may contribute. Compared with adults there are relatively few data on the prevalence of sleep-related obstructed breathing in children, most of them using questionnaires completed by their parents. In some studies from children 6 years old or younger, approximately $1 / 3$ were reported to snore occasionally and $10 \%-14 \%$ frequently [31]. It seems as older children snore less than younger. Witnessed apnoea has been reported in approximately $5 \%$ of children [32] but reliable estimates of the prevalence of OSA in children are difficult since interpretation of polysomnographic findings is controversial and based on scant normative data [33]. Also, the use of respiratory event counts such as the AHI may be inappropriate for childhood OSA, often characterized by prolonged obstructive hypoventilation with few apnoeic or hypopnoeic events. An interesting finding from epidemiological studies is that in contrast to adults, where OSA occurs primarily in males, in children it appears to occur equally between gender. It has been hypothesized that this is due to the lack of hormonal influences in the pre-pubertal child [34]. 


\subsection{Public Health and OSA}

For the public health relevance of OSA, it is important to understand the natural history of the syndrome and its association with host and environmental risk factors. This is useful for screening purposes and when evaluating the mechanisms that links OSA with chronic diseases. Cross sectional studies on prevalence do show effects of age, independently of the tendency of increased BMI with age. There are studies showing a doubling of AHI every 10 years [21,24]. When symptoms are included in the definition of OSA, the prevalence seems to fall above the age of 65 . The peak age of presentation is around 50 years and the prevalence falls off quite steeply after this age [31]. It is not known if this is because older patients simply do not complain about their symptoms or because OSA gives generally less adverse consequences compared to younger patients. In general, findings have demonstrated that the natural history of OSA includes a moderate level of progression, even in subjects not referred to sleep centres. There are longitudinally determined measures of AHI with age and BMI with larger changes observed in men compared to women. Middle age men with high BMI may experience the highest rate of increase in AHI over time and may therefore benefit most from prospective monitoring.

\section{OSA and Cardiovascular Disease}

Lately, OSA has gained a lot of attraction as a cardiovascular risk factor. OSA co-exists with other cardiovascular risk factors but has a strong independent association. Nowadays it is recommended for patients newly diagnosed with OSA that screening for cardiovascular risk, such as hypertension is undertaken [35]. Indeed, OSA has been increasingly implicated in the initiation and progression of several cardiovascular disorders such as coronary artery disease (CAD), cardiac arrhythmias, systemic hypertension, pulmonary hypertension, and stroke [36-40]. Circulatory physiology is changed in OSA and there are OSA related hypoxia, hypercapnia and blood pressure surges due to sympathetic over activation and acute imbalance of vasoactive hormones. These changes cannot only provoke acute coronary syndromes and stroke but if persistent also chronic consequences such heart failure [41].

The endothelium plays a key role in the pathophysiology of OSA and its impact on cardiovascular disease. Not only does it function as a separation between the blood and the vessel wall, the endothelium has a role in the maintenance of vascular tone, coagulation and cellular growth [41]. The precise mechanisms of endothelial dysfunction in OSA are not yet known but is believed that hypoxia, inflammation or oxidative stress initiate the process [42]. An example of this is that Nitric Oxide (NO) is a vasodilator secreted by the endothelium and a reduction in NO will subsequently lead to an impaired endothelial mediated vasodilatation.

OSA is also believed to cause increased oxidative "stress" which in turn plays a role in cardiovascular disease. Repeated hypoxic episodes cause intra-cellular structures to adapt to lower oxygen levels. Then, when the saturation level is increased during normal oxygen concentrations in the awake patient, these cells produce reactive oxygen species (ROS) which in turn have the potential to oxidize lipids and proteins. ROS also contribute to endothelial dysfunction, inflammation and ultimately atherosclerosis by augmenting NO breakdown [41].

Inflammation and metabolic dysfunction are known to have a strong impact on various vascular disorders. A raised Chain Reactive Protein (CRP) level reduces NO synthase (an enzyme that catalyses synthesis processes). NO is further reduced by hypoxia in OSA patients as described above. Hypoxia and sleep deprivation will additionally enhance the expression of inflammatory mediators such as interleukins and TNF $\alpha$. [43]. It has also been suggested that metabolic disturbances may link between OSA and cardiovascular disease. At least severe OSA correlates with increased insulin resistance. Subsequent high insulin levels also in non-obese patients have been reported to worsen with increasing AHI. One study showed that severe OSA was accompanied by a 5-fold increased risk of overt diabetes mellitus [44].

The vast majority of OSA patients (up to 95\%) has been reported to have hypertension. The actual mechanisms have been debated but cross sectional analyses suggest that OSA is an independent risk factor for high systolic as well as diastolic blood pressure [45]. There are several theories on the causative pathways of hypertension in OSA patients. Endothelial dysfunction and thereby impaired vasodilatation is one factor already mentioned. Additionally, hypoxia caused by apnoea/hyponoea increases sympathetic activity due to the episodes of arousal and subsequent fluctuations in catecholamine levels [46,47].

\section{Investigations and Diagnostics in OSA}

\subsection{Polysomnography}

The standard method to investigate and diagnose obstructive sleep apnoea is a polysomnography (PSG), also known as "sleep study". PSG is a multiparametric test used as a diagnostic tool in sleep medicine. The test result, displayed on a polysomnogram, is a comprehensive recording of the biophysiological changes that occur during sleep. The PSG monitor several body function including the respiratory and cardiovascular systems 
(pulse oximetry, ECG), brain (EEG), eye movement and muscle activity. This method will reveal which patients who have episodes of snoring, apnoea/hyponea and the frequency and lengths of these episodes. PSG, will, however, not identify the site of obstruction, nor will it give guidance in terms of treatment.

Diagnostic and radiological studies including lateral cephalometry, CT, MRI and endoscopy have been used to direct the treatment (e.g. surgical/non-surgical). These methods are all done when the patient is awake which gives only a limited characterization of obstruction during sleep.

\subsection{Lateral Cephalometric Radiographs}

One of the more common and inexpensive methods is the plain cephalometric radiograph, which may be used to assess both skeletal and soft tissue abnormalities. The drawback is that the pharyngeal area which indeed is a 3-dimensional structure, is investigated using a 2-dimensional method. Soft tissue can be identified, eg. tongue size and retro-glossal space. The retro-glossal space (also called posterior airway space, PAS), is meas- ured from the base of the tongue to the posterior pharyngeal wall. Skeletal measurements commonly involve measurements of the hyoid position and size and the position of the maxilla and mandible in relation to the skull base [48]. The distance from the mandibular base plane to the hyoid bone is most often the measured distance since it is known that inferior displacement of the hyoid leads to an increased degree of tongue base projection into the hypopharynx and lengthening of the airway, both contributing to airway collapse. With the use of lateral cephalometric analysis, the relations between the maxillofacial complex and the cranial base can be measured. There are several cephalometric measurements performed when planning orthognatic corrective surgery. For investigation of sleep apnoea, the most common points of measurement are: sella $(\mathrm{S})$, nasion $(\mathrm{N})$, anterior nasal spine (A) and supramentale (B) and the SNA and SNB angles represents the relative position of the maxilla and mandible to the skull base (Figure 1). It has been shown that OSA patients had smaller SNA and SNB angles (tendency of micrognathia) and displaced hyoid bones compared to non-OSA individuals [49]. Other

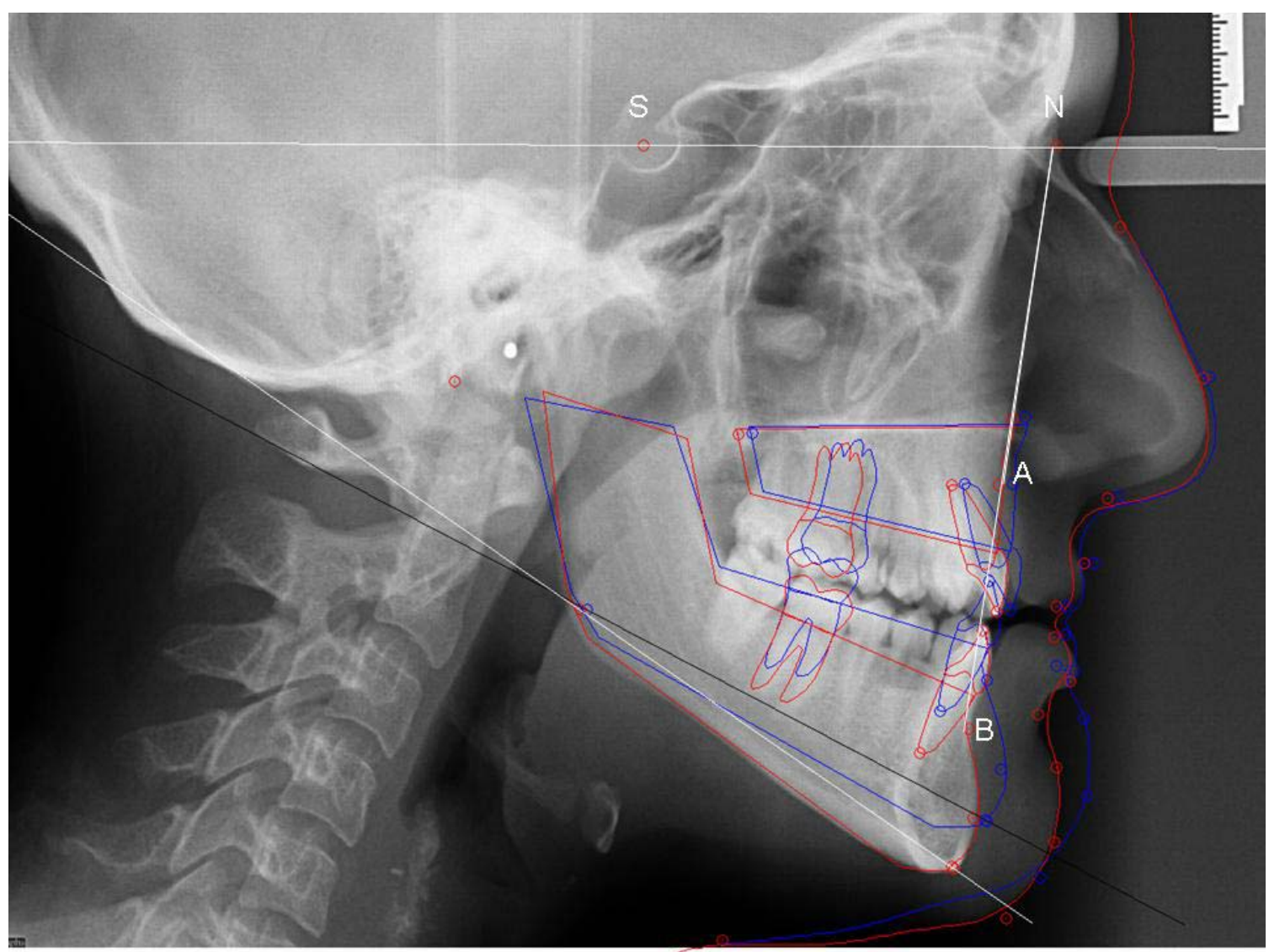

Figure 1. Cephalometry showing bimaxillary retrognatism in a patient with OSA. (S) sella, (N) nasion, (A) Down's A-point, subspinale, (B) Down's B-point, supramentale. SNA angle = maxillary anterior point in relation to sella-nasion axis and SNB angle = mandibular anterior point in relation to sella-nasion axis. Red lines show skeletal and soft tissue borders before surgery and blue lines the planned borders after bimaxillary advancement and anterior rotation. 
studies have distinguished two patient groups who have OSA [50,51]. One group comprises patients who are obese as measured by body mass index (BMI) and/or neck circumference and who have abnormal soft tissue measurements, previously described in the section on anatomical abnormalities and physiology of OSA. The other group are patients with normal BMI but who instead have skeletal abnormalities identified on cephalometric radiographs as a cause of their OSA. Ferguson and co-workers [51] evaluated neck circumference and found that individuals with OSA and normal neck size $(<42 \mathrm{~cm})$ had maxillofacial deformities, while individuals with larger neck size and OSA had larger tongues, longer mandibular-hyoid distance and elongated soft palate.

\subsection{Computed Tomography (CT)}

CT-scans offer a better precision than the plain film cephalometric radiograph. CT-scans are getting increasingly common and the technique is more or less standard in any preoperative planning of OSA patients. It does, however exhibit the same drawback as any other imaging method since the patient is awake while the investigation is performed and gives therefore the shape and dimension of the awake airway. Nevertheless, awake CT studies have shown narrowing of the oropharynx, especially in the retropalatal region in OSA patients compared with controls [52,53]. CT scans are also used to follow up surgical procedures such as uvulopalatopharyngeal plasty (UPPP) and maxillo-mandibular advancement.

\subsection{Magnetic Resonance Imaging (MRI)}

There are some obvious advantages with MRI; the patient is not exposed to radiation, soft tissues are well defined and with modern technologies, the dynamic airway can be evaluated with rapid image acquisition using multiple images per second. However, despite these advantages it has been difficult to use the technique during sleep and thus, truly evaluate OSA. There are a few studies on asleep patients and MRI scanning has shown differences between OSA and control patients, such as greater narrowing of velopharynx because of collapse of the lateral pharyngeal walls in OSA [54], but not to an extent to make MRI a sole diagnostic test [48]. Other studies have used MRI to analyse the anatomy of the suprahyoid muscles. For example, Schotland and coworkers [55] found more fat content in the genioglossus and geniohyoid muscles in OSA patients as compared to controls.

\subsection{Pharyngoscopy}

Endoscopy with fiberoptic equipment is a relatively inexpensive technique for evaluation of the airway in mul- tiple positions during both awake and asleep periods. Computer assisted airway measurements allows for an accurate mapping of the site of the airway obstruction. The technique has for example shown to have the ability to detect tongue base obstruction during sleep [56].

There are also other techniques to evaluate sleep apnoea and the level of obstruction, such as manometry, fluoroscopy and acoustic reflection but these will not be further discussed due to their relative unusualness.

\section{Treatment}

As in other medical disorders, life style changes should always be considered. Predisposing factors such as obesity, tonsilar hypertrophy and facial deformities should be corrected if possible. Intake of alcohol and sedatives should be avoided.

\subsection{Continuous Positive Airway Pressure (CPAP)}

CPAP has become the first line treatment option in OSA patients since it was introduced in 1981, by Sullivan and co-workers [57]. The equipment consists of a blower that generates positive pressure and it functions as a pneumatic splint preventing the upper airway from collapsing cyclically during sleep. The airway keeps open ("splint effect”) by increasing the intraluminal upper airway pressure above the pressure of the soft tissue airway structures. It will thereby improve nocturnal oxygenation, increase lung volume and the ventilatory drive [58,59]. CPAP is, as mentioned above considered the standard treatment for OSA, although concerns have been raised regarding the patient-CPAP interface (mask) and inadequate adherence and therefore, authors have criticised the method [58]. It has been suggested that firstly patients who have moderate to severe OSA $(\mathrm{AHI}>15)$ should be treated with CPAP [60]. Additionally, patients with mild OSA (AHI: 5 - 14) with co-morbidity, such as day time sleepiness, mood disorders or cardiovascular disease, could benefit from CPAP treatment. The recommendation of CPAP titration is that one starts at low pressure of $4-5 \mathrm{~cm} \mathrm{H} \mathrm{H}_{2} \mathrm{O}$, gradually increasing by $1-2 \mathrm{~cm}$ every 15 minutes until apnoeas/hypopnoeas disappear [58]. Usually the pressure has to be increased in supine position (as compared to lateral) and during REM sleep.

The long-term compliance using CPAP is dependent on side effects and patient motivation. The commonest side effects of CPAP are related to nasal symptoms of dryness, congestion and rhinorrhoea, which affect $25 \%$ $65 \%$ of the users [61]. Other side effects are sinusitis, conjunctivitis and pressure sore on the face from the mask. These side effects are if severe, all relative contraindications to CPAP and patient satisfaction and compliance should be monitored. 


\subsection{Surgery of the Palate and Oropharynx}

If the patient cannot or will not accept CPAP due to side effects and/or difficulties to sleep, surgery is an alternative. In the early 1980's the uvulopalatopharyngoplasty (UPPP/UP3) was first described as a method to surgically correct the upper airway [62]. UPPP is designed to eliminate palatal and pharyngeal redundancy by tonsillectomy + resection of excess mucosal and submucosal tissue in the pharyngeal area. Initially the technique was considered a great success but later, Sher and co-workers [63] reported only $40 \%$ success rate in achieving long term cure. In fact, other studies have described patients who worsened both objectively (PSG) and subjectively (increased day time sleepiness) after UPPP [64]. After this, patient selection has become increasingly important to identify appropriate candidates for the procedure and it has been suggested that patients who have the obstruction localized to the oropharynx would be the most suitable for UPPP. Studies have also shown that severity is not a prognostic factor for success or not. Patients with mild AHI classification can have a poor result with UPPP, while a candidate with severe disease can be fully cured [65]. Obesity is a negative prognostic factor and BMI > 40 is a contraindication to UPPP due to poor outcome of the procedure.

The main complications to UPPP are intra and post operative bleeding, airway obstruction during the initial postoperative period caused by significant oedema and dysphagia [66].

\subsection{Genioglossus and Hyoid Advancement}

The genioglossus advancement (GA) involves osteotomy of the chin (usually square shaped on the symphysis) to be able to move the geniotubercle with the genioglossus insertion forward without moving the mandible. This advancement places tension on the tongue musculature and thereby limits posterior displacement during sleep. The hyoid advancement (HA) technique implies an anterior movement of the hyoid bone by attaching it to the thyroid cartilage. The operation is usually performed in conjunction with GA or UPPP to reduce the severity of OSA. The success rates for GA and HA have been variable, ranging from $23 \%$ to $77 \%[67,68]$. In general, the associated surgical risks for these procedures are low, but include infection, oedema and dysphagia.

\subsection{Phase II Surgery}

Maxillomandibular advancement (MMA), also called Phase II surgery, expands the skeleton that encircles the airway and the entire airway is thereby enlarged. The operation consists of a bilateral osteotomy (Le Fort I osteotomy and bilateral sagittal split osteotomy, BSSO, of the mandible) and the maxillomandibular complexes are mobilized and diplaced anteriorly. Fixation is performed with titanium plates when a good occlusion is established. An advancement of $10-14 \mathrm{~mm}$ is usually recommended to achieve a successful improvement of the obstructed airways but a balanced aesthetic appearance is of course also important which means that phase II procedures suits patients with micrognathia best. However, interestingly enough, very few patients with pre-surgically ortognathic jaws are dissatisfied with their appearance after the advancement [69]. MMA is considered the most effective surgical sleep apnoea procedure currently available and the long-term success rate is usually over $90 \%$ [70,71]. Despite that bimaxillary surgery is considered a fairly invasive procedure, the associated surgical risks are low but includes bleeding, sensory disturbance of the inferior alveolar nerve and malocclusion.

\subsection{Oral Appliances}

The use of oral appliances to advance the mandible non-surgically is an increasingly popular method to treat OSA but has not the same effect on improving AHI compared to MMA surgery since the appliances advance the mandible only. It has been recommended in patients with primary snoring and mild to moderate OSA and has been shown to reduce AHI by $50 \%$ [72] which is considerably less than with the surgical method. A reason could be that the maxilla is not affected and that compliance may be low. Additionally, dental appliances can give temporomandibular joint disturbances, especially in long term use, due to stretching of ligaments around the disk capsule. A recent review of the literature also showed poor congruence in which design of the appliance that is most effective [73].

\section{Discussion and Guidelines for Treatment}

OSA has a multifactorial pathophysiology and is a significant public health problem with strong association with cardiovascular disease [35-47]. The incidence in the UK for example is for middle-age men $1 \%$ - $2 \%$ which is similar to diabetes mellitus type I and almost double that of severe asthma [74]. Corresponding figures for middle-age women in the UK are $0.5 \%-1 \%$. These figures are lower than comparable studies from other European centres, but the male:female ratio is similar $[28,29]$. If cardiovascular disease is the long term consequence of OSA, the shorter perspective effects are interrupted sleep, day time sleepiness, impaired cognition, mood and even personality changes [75]. This in turn, will have consequences on alertness during e.g. driving and at work which may lead to work and traffic related accidents. It has been speculated that road traffic accident risk increases with up to 12 times in untreated OSA patients [76]. 
Non-surgical treatment is the first line option together with life style changes when treating patients with OSA. CPAP has been established as the treatment with the firmest evidence base [77]. Randomized control trials comparing oral appliances to CPAP are all crossover studies conducted in patients with symptomatic OSA. For polysomnographic outcomes, the mean values for AHI, oxygen saturation and sleep fragmentation favour CPAP in these studies [78,79]. For surgical interventions, the number and qualities of randomized control trails are poor and case series on UPPP suggest at best $50 \%$ improvement in $50 \%$ of the cases and is not recommended as a first line treatment $[80,81]$. Maxillomandibular advancement has shown promising long-term results in cohort studies [66-71] but randomised controlled trials are still required.

\section{Conclusions}

Sleep apnoea is formally defined as an apnoea-hypopnoea index (AHI) of at least 5 episodes/hour (mild). Contributing factors are obesity, alcohol and sedatives contribute, as well as tonsilar hypertrophy and mandibular retrognathia (retroglossal space limited). Endocrine conditions such as hypothyroidism and acromegaly have also been reported associated with OSA. Except for excessive daytime sleepiness, headaches and irritability, OSA may lead to hypertension, heart failure, MI and stroke. Sleepiness may also lead to work and road traffic related accidents.

Management involves reduction of predisposing factors such as obesity and alcohol. Treatment with continuous positive airway pressure (CPAP) is currently most common. Also uvulopalatopharyngeal surgery and so called phase II surgery, which means advancement of the jaw(s) with orthognatic surgical methods are used. Treatment with dental appliances is getting increasingly popular but the long term outcome remains debatable.

\section{REFERENCES}

[1] B. T. Woodson and R. Franco, "Physiology of Sleep Disordered Breathing," Otolaryngologic Clinics of North America, Vol. 40, No. 4, 2007, pp. 691-711. doi:10.1016/j.otc.2007.04.002

[2] E. D. Weitzman, C. P. Pollak, B. B. Borowiecki, B. Burack, R. Sphrintzen and S. Rakoff, “The HypersomniaSleep Apnea Syndrome: Site and Mechanism of Upper Airway Obstruction," Transactions of the American Neurological Association, Vol. 102, 1977, pp. 150-153.

[3] C. Guilleminault, M. Hill, F. B. Simmons, N. Powel, R. Riley and R. Stoohs, "Passive Constriction of the Upper Airway during Central Apneas: Fiberoptic and EMG Investigation," Respiration Physiology, Vol. 108, No. 1, 1997, pp. 11-22. doi:10.1016/S0034-5687(97)02529-2

[4] J. E. Remmers, W. J. de Groot, E. K. Sauerland and A. M.
Anch, "Pathogenesis of Upper Airway Occlusion during Sleep,” Journal of Applied Physiology, Vol. 44, No. 6, 1978, pp. 931-938.

[5] C. Guilleminault, R. Stoohs, T. Shiomi, C. Kushida and I. Schnittger, "Upper Airway Resistance Syndrome, Nocturnal Blood Pressure Monitoring and Borderline Hypertension," Chest, Vol. 109, No. 4, 1996, pp. 901-908. doi:10.1378/chest.109.4.901

[6] S. Isono, J. Remmers, A. Tanaka, Y. Sho, J. Sato and T. Nishino, "Anatomy of the Pharynx in Patients with Obstructive Sleep Apnoea and in Normal Subjects,” Journal of Applied Physiology, Vol. 82, No. 4, 1997, pp. 13191326.

[7] M. Zucconi, A. Caprioglio, G. Calori, L. Ferini-Strambi, A. Oldani, C. Castronovo and S. Simme, "Craniofacial Modifications in Children with Habitual Snoring and Obstructive Sleep Apnoea: A Case Control Study," The European Respiratory Journal, Vol. 13, No. 2, 1999, pp. 411-417. doi:10.1183/09031936.99.13241199

[8] J. A. Dempsy, J. B. Skatrud, A. J. Jacques, S. J. Ewanowski, B. T. Woodson, P. R. Hanson and B. Goodman, "Anatomical Determinations of Sleep Disordered Breathing across the Spectrum of Clinical and NonClinical Subjects," Chest, Vol. 122, No. 3, 2002, pp. 840 851. doi:10.1378/chest.122.3.840

[9] D. O. Rodenstein, G. Dooms, Y. Thomas, G. Liistro, D. C. Stanscu, C. Culée and G. Aubert-Tulkens, "Pharyngeal Shape and Dimensions in Healthy Subjects, Snorers and Patients with Obstructive Sleep Apnoea," Thorax, Vol. 45, No. 10, 1990, pp. 722-727.

[10] B. T. Woodson, "Expiratory Pharyngeal Airway Obstruction during Sleep: A Multiple Element Model,” Laryngoscope, Vol. 113, No. 9, 2003, pp. 1450-1459. doi:10.1097/00005537-200309000-00004

[11] J. C. Leiter, "Upper Airway Shape. Is It Important in the Pathogenesis of Obstructive Sleep Apnea?" American Journal of Respiratory and Critical Care Medicine, Vol. 153, No. 3, 1996, pp. 894-898.

[12] S. Tuck and J. Remmers, "Mechanical Properties of the Passive Pharynx in Vietnamese Pot-Bellied Pigs. II. Dynamics," Journal of Applied Physiology, Vol. 92, 2002, pp. 2236-2244.

[13] W. Van De Graaff, "Thoracic Traction on the Trachea: Mechanisms and Magnitude,” Journal of Applied Physiology, Vol. 70, 1991, pp. 1328-1336.

[14] A. R. Schwatz, S. P. Patil, A. M. Laffan, et al., "Obesity and Obstructive Sleep Apnoea," Proceedings of the American Thoracic Society, Vol. 5, No. 2, 2008, pp. 185-192. doi:10.1513/pats.200708-137MG

[15] S. Margetic, C. Gazzola, G. G. Pegg, et al., "Leptin: A Review of Its Peripheral Actions and Interactions," International Journal of Obesity, Vol. 26, No. 11, 2002, pp. 1407-1433. doi:10.1038/sj.ijo.0802142

[16] C. G. Tankersley, C. O’Donnell, M. J. Daood, et al., "Leptin Attenuates Respiratory Complications Associated with the Obese Phenotype," Journal of Applied Physiology, Vol. 85, 1998, pp. 2261-2269.

[17] A. S. M. Shamsuzzaman, B. J. Gersh and V. K. Somers, 
"Obstructive Sleep Apnea Implications for Cardiac and Vascular Disease," Journal of the American Medical Association, Vol. 290, No. 14, 2003, pp. 1906-1914. doi:10.1001/jama.290.14.1906

[18] E. Lugaresi and G. Plazzi, "Heavy Snorer Disease: From Snoring to Sleep Apnoea Syndrome: An Overview,” Respiration, Vol. 64, 1997, pp. 11-14. doi:10.1159/000196730

[19] L. Ferini-Strambi, M. L. Fantini and C. Castronovo, "Epidemiology of Obstructive Sleep Apnea Syndrome," Minerva Medica, Vol. 95, 2004, pp. 187-202.

[20] E. Bixler, A. Vgontzas, T. Ten Have, et al., "Effects of Age on Sleep Apnoea in Men,” American Journal of Respiratory and Critical Care Medicine, Vol. 157, No. 1, 1998, pp. 144-148.

[21] E. Bixler, A. Vgontzas, T. Ten Have, et al., "Prevalence of Sleep Disordered Breathing in Women," American Journal of Respiratory and Critical Care Medicine, Vol. 163, No. 3, 2001, pp. 608-613.

[22] C. J. Durán, S. Esnaola, R. Rubio and A. Iztueta, "Obstructive Sleep Apnea-Hypopnea and Related Clinical Features in a Population-Base Sample of Subjects Aged 30 to 70 yr," American Journal of Respiratory and Critical Care Medicine, Vol. 163, 2001, pp. 685-689.

[23] C. J. Durán, "Prevalence of Obstructive Sleep ApneaHypopnea and Related Clinical Features in the Elderly: A Population-Based Study in the General Population Aged 71 - 100,” Abstract: Sleep Odyssey, Montevideo, 2001.

[24] T. Young, E. Shahar, F. J. Nieto, et al., "Predictors of Sleep-Disordered Breathing in Community Dwelling Adults: The Sleep Heart Health," Archives of Internal Medicine, Vol. 216, 2002, pp. 893-900. doi:10.1001/archinte.162.8.893

[25] R. P. Millman, C. C. Carlisle, S. T. McGarvey, et al., "Body Fat Distribution and Sleep Apnea Severity in Women,” Chest, Vol. 107, No. 2, 1995, pp. 362-366. doi:10.1378/chest.107.2.362

[26] T. Young, M. Palta, J. Dempsey, et al., "The Occurrence of Sleep-Disordered Breathing among Middle-Aged Adults," New England Journal of Medicine, Vol. 328, 1993, pp. 1230-1235. doi:10.1056/NEJM199304293281704

[27] L. G. Olson, M. T. King, M. G. Hensley, et al., "A Community Study of Snoring and Sleep-Disordered Breathing," American Journal of Respiratory and Critical Care Medicine, Vol. 152, No. 2, 1995, pp. 707-710.

[28] L. Ferini-Strambi, M. Zucconi, V. Castronov, et al., "Snoring and Sleep Apnea: A Population Study in Italian Women,” Sleep, Vol. 22, 1999, pp. 859-864.

[29] L. Ferini-Strambi, M. Zucconi, S. Palazzi, et al., "Snoring and Nocturnal Oxygen Desaturations in an Italian Middle-Aged Male Population: Epidemiologic Study with an Ambulatory Device," Chest, Vol. 152, No. 2, 1994, pp. 1759-1764. doi:10.1378/chest.105.6.1759

[30] C. O’Connor, K. S. Thgornley and P. J. Hanly, “Gender Differences in the Polysomnographic Features of Obstructive Sleep Apnea,” American Journal of Respiratory and Critical Care Medicine, Vol. 161, 2000, pp. 14651472.
[31] L. Ferini-Strambi, M. L. Fantini and C. Castronovo, "Epidemiology of Obstructive Sleep Apnea Syndrome,” Minerva Medicine, Vol. 95, 2004, pp. 187-202.

[32] N. J. Ali, D. J. Pitson and J. R. Strandling, "Snoring, Sleep Distrubance and Behavior in 4 - 5 Year Olds," Archives of Disease in Childhood, Vol. 68, No. 3, 1993, pp. 360-366. doi:10.1136/adc.68.3.360

[33] J. P. Tang, C. L. Rosen, E. K. Larkin, et al., "Identification of Sleep-Disordered Breathing in Children: Variation with Event Definition,” Sleep, Vol. 25, 2002, pp. 72-79.

[34] T. Young, P. E. Peppard and D. J. Gottlieb, “Epidemiology of Obstructive Sleep Apnea: Apopulation Health Perspective," American Journal of Respiratory and Critical Care Medicine, Vol. 165, No. 9, 2002, pp. 1217-1239. doi:10.1164/rccm.2109080

[35] A. V. Chobanian, G. L. Bakris, H. Black, et al., "Seventh Report of the Joint National Committee on Prevention, Detection, Evaluation and Treatment of High Blood Pressure,” Hypertension, Vol. 42, 2003, pp. 1206-1252. doi:10.1161/01.HYP.0000107251.49515.c2

[36] E. Shahar, C. W. Whitney, E. T. Redline, et al., "Sleep Disordered Breathing and Cardiovascular Disease: Cross Sectional Results of Sleep Heart Health Study,” American Journal of Respiratory and Critical Care Medicine, Vol. 163, 2001, pp. 19-25.

[37] V. Hoffstein and S. Mateika, "Cardiac Arrhytmias, Snoring and Sleep Apnea,” Chest, Vol. 106, No. 2, 1994, pp. 466-471. doi:10.1378/chest.106.2.466

[38] C. T. Worsnop, M. T. Naughton, C. E. Barter, et al., “The Prevalence of Obstructive Sleep Apnea in Hypertensives," American Journal of Respiratory and Critical Care Medicine, Vol. 157, 1998, pp. 111-115.

[39] B. M. Sanner, C. Doberauer, M. Konermann, et al., "Pulmonary Hypertension in Patients with Obstructive Sleep Apnea Syndrome," Archives of Internal Medicine, Vol. 157, 1997, pp. 2483-2487. doi:10.1001/archinte.157.21.2483

[40] M. E. Dyken, V. K. Somers, T. Yamada, et al., "Investigating the Relationship between Stroke and Obstructive Sleep Apnea,” Stroke, Vol. 27, 1996, pp. 401-407. doi:10.1161/01.STR.27.3.401

[41] M. Butt, G. Dwivedi, O. Kahir and G. Lip, "Obstructive Sleep Apnea and Cardiovascular Disease,” International Journal of Cardiology, Vol. 139, No. 1, 2010, pp. 7-16. doi:10.1016/j.ijcard.2009.05.021

[42] S. K. Venugopal, S. Devaraj, I. Yuhanna, et al., "Demonstration that C-Reactive Protein Decreases eNOS Expression and Bioactivity in Human Aortic Endothelial Cells," Circulation, Vol. 106, 2002, pp. 1439-1441. doi:10.1161/01.CIR.0000033116.22237.F9

[43] A. N. Vgontzas, D. A. Papanicolaou, E. O. Bixler, et al., "Elevation of Plasma Cytokines in Disorders of Excessive Daytime Sleepiness: Role of Sleep Disturbance and Obesity,” Journal of Clinical Endocrinology \& Metabolism, Vol. 82, No. 5, 1997, pp. 1313-1316. doi:10.1210/jc.82.5.1313

[44] K. Spiegel, R. Leproult and E. Van Cauter, "Impact on Sleep Dept on Metabolic and Endocrine Function,” Lancet, Vol. 354, No. 9188, 1999, pp. 1435-1439. 


\section{doi:10.1016/S0140-6736(99)01376-8}

[45] P. Lavie, P. Herer and V. Hoffstein, “Obstructive Sleep Apnoea as a Risk Factor for Hypertension: Population Study," British Medical Journal, Vol. 320, 2000, pp. 479482. doi:10.1136/bmj.320.7233.479

[46] O. Marrone, L. Riccobono, A. Salvaggio, et al., "Catecholamines and Blood Pressure in Obstructive Sleep Apnoea Syndrome,” Chest, Vol. 103, No. 3, 1993, pp. 722727. doi:10.1378/chest.103.3.722

[47] J. T. Carlson, J. Hedner, M. Elam, et al., “Augmented Resting Sympathetic Activity in Awake Patients with Sleep Apnoea," Chest, Vol. 103, No. 6, 1993, pp. 17631768. doi:10.1378/chest.103.6.1763

[48] K. Thakkar and M. Yao, "Diagnostic Studies in Obstructive Sleep Apnea," Otolaryngologic Clinics of North America, Vol. 40, No. 4, 2007, pp. 785-805. doi:10.1016/j.otc.2007.04.005

[49] B. deBerry-Borwiecki, A. Kukwa and R. H. Blanks, "Cephalometric Analysis for Diagnosis and Treatment of Obstructive Sleep Apnoea,” Laryngoscope, Vol. 98, 1988, pp. 226-234.

[50] M. Tsuchiya, A. A. Lowe, E. K. Pae, et al., "Obstructive Sleep Apnoea Subtypes by Cluster Analysis,” American Journal of Orthodontics \& Dentofacial Orthopedics, Vol. 101, No. 6, 1992, pp. 533-542. doi:10.1016/0889-5406(92)70128-W

[51] K. A. Ferguson, T. Ono, A. A. Lowe, et al., "The Relationship between Obesity and Craniofacial Structure in Obstructive Sleep Apnoea,” Chest, Vol. 108, No. 2, 1995, pp. 375-381. doi:10.1378/chest.108.2.375

[52] M. E. Bohlman, E. F. Haponik, P. L. Smith, et al., “CT Demonstration of Pharyngeal Narrowing in Obstructive Sleep Apnea," American Journal of Roentgenology, Vol. 140, 1983, pp. 543-548.

[53] E. F. Haponik, P. L. Smith, M. E. Bohlman, et al., "Computerized Tomography in Obstructive Sleep Apnea. Correlation of Airway Size with Physiology during Sleep and Wakefulness," American Review of Respiratory Disease, Vol. 127, No. 2, 1983, pp. 221-226.

[54] M. A. Cicar, G. Juan, V. Martinez, et al., "Magnetic Resonance Imagning of the Pharynx in OSA Patients and Healthy Subjects," European Respiratory Journal, Vol. 17, No. 1, 2001, pp. 79-86. doi:10.1183/09031936.01.17100790

[55] H. M. Schotland, E. K. Insko and R. J. Schwab, “Quantitative Magnetic Resonance Imaging Demonstrates Alterations of the Lingual Musculature in Obstructive Sleep Apnoea,” Sleep, Vol. 22, No. 5, 1999, pp. 605-613.

[56] B. T. Woodson and M. R. Wooten, "Comparison of Upper-Airway Evaluations during Wakefulness and Sleep,” Laryngoscope, Vol. 104, No. 7, 1994, pp. 821-828. doi:10.1288/00005537-199407000-00007

[57] C. E. Sulivan, F. G. Issa, M. Berthon-Jones, et al., "Reversal of Obstructive Sleep Apnoea by Continous Positive Airway Pressure Applied through Nares," Lancet, Vol. 317, No. 8225, 1981, pp. 862-865. doi:10.1016/S0140-6736(81)92140-1

[58] S. Chowdhuri, "Continuous Positive Airway Pressure for the Treatment of Sleep Apnoea," Otolaryngologic Clinics of North America, Vol. 40, No. 4, 2007, pp. 807-827. doi:10.1016/j.otc.2007.04.011

[59] S. M. T. Moura, L. R. Bittencourt and M. C. Bagnato, "Acute Effect of Nasal Continuous Positive Air Pressure on the Ventilator Control of Patients with Obstructive Sleep Apnoea,” Respiration, Vol. 68, 2001, pp. 243-249.

[60] D. I. Loube, P. C. Gay, K. P. Strohl, et al., "Indications for Positive Airway Pressure Treatment of Adult Obstructive Sleep Apnea Patients: A Consensus Statement," Chest, Vol. 115, No. 3, 1999, pp. 863-866. doi:10.1378/chest.115.3.863

[61] J. L. Pepin, P. Leger, D. Veale, et al., "Side Effects of Nasal Continuous Positive Airway Pressure in Sleep Apnea Syndrome. Study of 193 Patients in Two French Sleep Centres," Chest, Vol. 107, No. 2, 1995, pp. 375-381. doi:10.1378/chest.107.2.375

[62] S. Fujita, W. Conway, F. Zorick, et al., "Surgical Correction of Anatomic Abnormalities in Obstructive Sleep Apnea Syndrome: Uvulopalatopharyngoplasty," Otolaryngology-Head and Neck Surgery, Vol. 89, 1981, pp. 923-934.

[63] A. E. Sher, K. B. Schechtman and J. F. Piccirilollo, "The Efficacy of Surgical Modifications of the Upper Airways in Adults with Obstructive Sleep Apnea,” Sleep, Vol. 19, 1996, pp. 156-177.

[64] B. A. Senior, L. Rosenthal, A. Lumley, et al., "Efficacy of Uvulopalatopharyngoplasty in Unselected Patients with Mild Obstructive Sleep Apnoea," Otolaryngology-Head and Neck Surgery, Vol. 123, No. 3, 2000, pp. 179-182. doi:10.1067/mhn.2000.106707

[65] M. Friedman, R. Vidysagar, D. Bliznikas, et al., "Does Severity of Obstructive Sleep Apnea/Hypopnea Syndrome Predict Uvulopalatopharyngeal Plasty Outcome?" Lanryngoscope, Vol. 115, No. 12, 2005, pp. 2109-2113. doi:10.1097/01.MLG.0000181505.11902.F7

[66] M. Friedman and P. Schalch, "Surgery of the Palate and Oropharynx," Otolaryngologic Clinics of North America, Vol. 40, No. 4, 2007, pp. 829-843. doi:10.1016/j.otc.2007.04.010

[67] K. K. Li, N. B. Powell, R. W. Riley, et al., "Overwiev of Phase I Surgery for Obstructive Sleep Apnoea Syndrome," Ear, Nose \& Throat Journal, Vol. 78, 1999, pp. 836-845.

[68] P. P. Hsu and R. H. Brett, "Multiple Level Pharyngeal Surgery for Obstructive Sleep Apnoea," Singapore Medical Journal, Vol. 42, 2001, pp. 160-164.

[69] K. K. Li, R. W. Riley, N. B. Powell, et al., "Patient's Perception of the Facial Appearance after Maxillomandibular Advancement for Obstructive Sleep Apnoea Syndrome," Journal of Oral and Maxillofacial Surgery, Vol. 59, No. 4, 2001, pp. 377-380. doi:10.1053/joms.2001.21870

[70] K. K. Li, N. B. Powell, R. W. Riley, et al., "Long-Term Results of Maxillomandibular Advancement Surgery," Sleep Breath, Vol. 4, No. 3, 2000, pp. 137-139. doi:10.1055/s-2000-11564

[71] R. Conradt, W. Hochban, U. Brandenburg, et al., "Long 
Term Results after Surgical Treatment of Obstructive Sleep Apnoea by Maxillomandibular Advancement," European Respiratory Journal, Vol. 10, No. 1, 1997, pp. 123-128. doi:10.1183/09031936.97.10010123

[72] A. Padme, N. Ramakrisham and V. Narayanas, "Management of Obstructive Sleep Apnoea: A Dental Perspective," Indian Journal of Dental Research, Vol. 18, No. 4, 2007, pp. 201-209. doi:10.4103/0970-9290.35833

[73] A. Ahrens, C. McGrath and U. Hägg, “A Systematic Review of the Efficacy of Oral Appliances in the Management of Obstructive Sleep Apnea," European Journal of Orthodontics, Vol. 13, 2011.

[74] T. Young, M. Palta, J. Dempsey, et al., "The Occurrence of Sleep-Disordered Breathing among Middle-Aged Adults,” New England Journal of Medicine, Vol. 328, 1993, pp. 1230-1235. doi:10.1056/NEJM199304293281704

[75] H. M. Enleman, S. E. Martin and N. J. Douglas, "Effect of Continous Positive Airway Pressure Treatment on Daytime Function in Sleep Apnoea/Hyponoea Syndrome," Lancet, Vol. 343, No. 8897, 1994, pp. 572-575. doi:10.1016/S0140-6736(94)91522-9

[76] J. Teran-Santos, A. Jimenez-Gomez and J. CorderoGueura, "The Association between Sleep Apnoea and Risk of Traffic Accidents," New England Journal of
Medicine, Vol. 340, 1999, pp. 847-851. doi:10.1056/NEJM199903183401104

[77] J. Wright, J. White and F. Duchane, "Continous Positive Arway Pressure for Obstructive Sleep Apnoea," The Cochrane Library, No. 1, 2002.

[78] H. M. Engleman, J. P. McDonald, D. Graham, et al., "Randomized Crossover Trial of Two Treatments for Sleep Apnoea/Hyponoea Syndrome: Continous Positive Airway Pressure and Mandibular Repositioning Splint," American Journal of Respiratory and Critical Care Medicine, Vol. 166, No. 6, 2002, pp. 855-859. doi:10.1164/rccm.2109023

[79] Y. K. Tan, P. R. L’Éstrange, Y. M. Luo, et al., "Mandibular Advancement Splints and Continous Positive Airway Pressure in Patients with Obstructive Sleep Apnoea: A Randomized Crossover Trial,” European Journal of Orthodontics, Vol. 24, No. 3, 2002, pp. 239-249. doi:10.1093/ejo/24.3.239

[80] A. E. Sher, K. B. Schechtman and J. F. Piccirillo, "The Efficacy of Surgical Modifications of the Upper Airway in Adults with Obstructive Sleep Apnoea Syndrome," Sleep, Vol. 19, No. 2, 1996, pp. 156-177.

[81] S. A. Bridgman, K. M. Dunn and F. Ducharme, "Surgery for Obstructive Sleep Apnoea," The Cochrane Library, No. 1, 2002. 